\title{
Coupled diffusion and phase transition: Phase fields, constraints, and the Cahn-Hilliard equation
}

\author{
Fernando P. Duda $\cdot$ Adel F. Sarmiento $\cdot$ Eliot Fried $\mathbb{D}$
}

Received: 12 September 2020/Accepted: 25 February 2021 / Published online: 3 May 2021

(C) The Author(s) 2021

\begin{abstract}
We develop a constrained theory for constituent migration in bodies with microstructure described by a scalar phase field. The distinguishing features of the theory stem from a systematic treatment and characterization of the reactions needed to maintain the internal constraint given by the coincidence of the mass fraction and the phase field. We also develop boundary conditions for situations in which the interface between the body and its environment is structureless and cannot support constituent transport. In addition to yielding a new derivation of the CahnHilliard equation, the theory affords an interpretation of that equation as a limiting variant of an Allen-Cahn type diffusion system arising from the unconstrained theory obtained by considering the mass fraction and the phase field as independent quantities. We corroborate that interpretation with three-dimensional
\end{abstract}

\section{F. P. Duda}

Programa de Engenharia Mecânica, Programa de Engenharia de Nanotecnologia, COPPE, Universidade Federal do Rio de Janeiro, Cidade Universitária, Rio de Janeiro CEP 21941-972, Brazil

e-mail: duda@mecanica.ufrj.br

\section{A. F. Sarmiento · E. Fried $(\bowtie)$}

Mathematics, Mechanics and Materials Unit, Okinawa Institute of Science and Technology Graduate University, 1919-1 Tancha, Onna, Okinawa, Japan

e-mail: eliot.fried@oist.jp

A. F. Sarmiento

e-mail: adelsarmiento@gmail.com numerical simulations of a recently proposed benchmark problem.

Keywords Microforce balance $\cdot$ Free-energy imbalance $\cdot$ Allen-Cahn theory $\cdot$ Regularization . Phase separation

\section{Introduction}

In this paper, we are concerned with continuum theories for constituent migration in bodies with microstructure described by a scalar phase field. We consider two different constitutive theories according to which the concentration and the phase field are either constrained to be equal or are independent. These considerations give rise to theories of the CahnHilliard (Cahn and Hilliard [1]) type and of the AllenCahn (Allen and Cahn [2]) type, respectively. Although they share the same basic principles, the theories differ at the constitutive level. This leads to an interpretation of the constrained theory underlying the Cahn-Hilliard family as a limiting variant of the unconstrained theory underlying the Allen-Cahn family. Our approach to the constrained case relies on a careful treatment and characterization of the reactions needed to maintain the internal constraint.

To the extent that the phase field is assumed to coincide with the constituent concentration, our 
pathway to the Cahn-Hilliard equation is in line with all previous continuum mechanical derivations, including the original one advanced by Gurtin [3] and subsequent alternatives due to Miranville [4], Fried and Sellers [5], Podio-Guidugli [6], Morro [7], and Heida et al. [8]. In a departure from those derivations, we treat the coincidence between the phase field and the constituent concentration as an internal constraint that is maintained by appropriate reactions. The presence and implications of this constraint have been overlooked in all previous continuum mechanical derivations. In recognition of the prominent importance of constrained materials in continuum mechanics, we believe that an explicit consideration of the internal constraints that underpin certain phase-field models can provide important insights. Support for this belief is provided by recent studies of Duda et al. [9, 10], who exploit a phase-field theory for irreversible fracture, due to da Silva et al. [11], in which an internal constraint is used to prohibit the spurious healing of cracks which would otherwise be allowed.

The remainder of this paper is organized as follows. First, in Sect. 2, we introduce the basic notions and laws necessary to describe constituent migration in continuum bodies with microstrucre described by a scalar phase field. In Sect. 3, we introduce the constitutive assumptions needed to obtain theories of the Cahn-Hilliard type. In Sect. 4, we address theories of the Allen-Cahn type coupled with diffusion and observe that one such theory can be identified as a regularization of the Cahn-Hilliard equation. In Sect. 5, we develop boundary conditions contingent on the simplifying assumption that the interface between the body and the surrounding environment is a structureless surface within which constituent transport can be neglected. Furthermore, we restrict attention to uncoupled zero-dissipation conditions. Finally, in Sect. 6, we use numerical simulations of a benchmark problem to corroborate the relation between the Cahn-Hilliard equation and the coupled Allen-Cahn diffusion system presented in Sect. 4.

\section{Basic notions}

In this section, we introduce the basic laws that govern the problem of constituent transport in a two- component body with microstructure. The state of the body, hereafter identified with a fixed region $\mathcal{B}$ of three-dimensional Euclidean point space, is described by the mass fraction $c$ of one of its components, while its microstructure is described by a phase field $\varphi$. Following Fried and Gurtin [12], the latter field is viewed as an independent kinematical descriptor. The basic laws-namely the constituent content balance, the microforce balance, and the free-energy imbalance-that govern the behavior of $\mathcal{B}$ are introduced next.

2.1 Constituent content and microforce balances

The constituent content balance states that

$\frac{\mathrm{d}}{\mathrm{d} t} \int_{\mathcal{P}} c \mathrm{~d} v=-\int_{\partial \mathcal{P}} \boldsymbol{J} \cdot \boldsymbol{n} \mathrm{d} a+\int_{\mathcal{P}} m \mathrm{~d} v$

for every part $\mathcal{P}$ of $\mathcal{B}$, where $\boldsymbol{\jmath}$ and $m$ are the constituent flux and supply, respectively. Using standard arguments, we arrive at the pointwise constituent content balance:

$\dot{c}=-\operatorname{div} \boldsymbol{\jmath}+m$.

Consistent with the interpretation of the phase field $\varphi$ as an indepedent kinematical descriptor, we introduce the microforce balance, which requires that

$\int_{\partial \mathcal{P}} \boldsymbol{\xi} \cdot \boldsymbol{n} \mathrm{d} a+\int_{\mathcal{P}}(\pi+\gamma) \mathrm{d} v=0$

for every part $\mathcal{P}$ of $\mathcal{B}$, where $\xi$ is the microstress vector, and $\pi$ and $\gamma$ the internal and external microforce densities. The corresponding pointwise version is

$\operatorname{div} \xi+\pi+\gamma=0$.

\subsection{Free-energy imbalance}

The free-energy imbalance represents a mechanical version of the first and second laws of thermodynamics and in the present context requires that

$$
\begin{aligned}
\frac{\mathrm{d}}{\mathrm{d} t} \int_{\mathcal{P}} \psi \mathrm{d} v \leq & \int_{\partial \mathcal{P}}(\boldsymbol{\xi} \dot{\varphi}-\mu \boldsymbol{\jmath}) \cdot \boldsymbol{n} \mathrm{d} a \\
& +\int_{\mathcal{P}}(\mu m+\gamma \dot{\varphi}) \mathrm{d} v
\end{aligned}
$$


for every part $\mathcal{P}$ of $\mathcal{B}$, where $\psi$ is the free-energy density and $\mu$ is the constituent chemical potential. The corresponding pointwise form reads

$$
\dot{\psi}-\mu \dot{c}+\pi \dot{\varphi}-\xi \cdot \operatorname{grad} \dot{\varphi}+\zeta \cdot \boldsymbol{\jmath} \leq 0,
$$

where $\zeta$ denotes the gradient of the chemical potential:

$$
\zeta=\operatorname{grad} \mu \text {. }
$$

\section{Cahn-Hilliard type theory}

We next derive a generalization of the Cahn-Hilliard equation. In line with the approach of Gurtin [3], the derivation is predicated on the assumption that the mass fraction $c$ must coincide with the phase field $\varphi$. Contrary to the classical approach, that coincidence is given the status of an internal constraint and treated accordingly. Our treatment follows ideas pioneered by Capriz [13].

\subsection{Constraint and its implications}

We assume that the mass fraction $c$ must coincide with the phase field $\varphi$ :

$c=\varphi$.

Our interpretation of (8) as an internal constraint entails the decomposition of the chemical potential $\mu$, the internal microforce $\pi$, the microstress $\xi$, and the chemical potential gradient $\zeta$ into active and reactive components:

$$
\left.\begin{array}{l}
\mu=\mu_{a}+\mu_{r}, \\
\pi=\pi_{a}+\pi_{r}, \\
\xi=\xi_{a}+\xi_{r}, \\
\zeta=\zeta_{a}+\zeta_{r}
\end{array}\right\}
$$

In keeping with standard practice, we assume that $\mu_{r}$, $\pi_{r}, \xi_{r}$ cannot expend power and that $\boldsymbol{\zeta}_{r}$ cannot produce dissipation. Consequent to this requirement, the equality

$$
\left(\pi_{r}-\mu_{r}\right) \dot{\varphi}-\boldsymbol{\xi}_{r} \cdot \operatorname{grad} \dot{\varphi}+\zeta_{r} \cdot \boldsymbol{\jmath}=0
$$

must hold for all choices of $\dot{\varphi}, \operatorname{grad} \dot{\varphi}$, and $\boldsymbol{\jmath}$. Moreover, by (8) and (10), the pointwise form (6) of the free-energy imbalance simplifies to $\dot{\psi}-\left(\mu_{a}-\pi_{a}\right) \dot{\varphi}-\boldsymbol{\xi}_{a} \cdot \nabla \dot{\varphi}+\zeta_{a} \cdot \boldsymbol{\jmath} \leq 0$.

From (11), we see that $\mu_{a}-\pi_{a}$ and $\zeta_{a}$ are powerconjugate to $\dot{\varphi}$ and $\nabla \dot{\varphi}$, respectively, and that $\zeta_{a}$ is dissipation conjugate to $\boldsymbol{\jmath}$. Since it is possible to construct a process in which $\dot{\varphi}, \operatorname{grad} \dot{\varphi}$, and $\boldsymbol{\jmath}$ can be chosen independently at any arbitrary point in space and instant of time, we infer from (10) that $\mu_{r}=\pi_{r}$, $\boldsymbol{\xi}_{r}=\mathbf{0}$, and $\zeta_{r}=\mathbf{0}$ or, equivalently, that

$\mu_{a}-\pi_{a}=\mu-\pi, \quad \xi_{a}=\xi, \quad$ and $\quad \zeta_{a}=\zeta$.

Combining (10) and (11), we arrive a reduced version,

$\dot{\psi}-(\mu-\pi) \dot{\varphi}-\xi \cdot \operatorname{grad} \dot{\varphi}+\boldsymbol{\zeta} \cdot \boldsymbol{\jmath} \leq 0$,

of the pointwise free-energy imbalance.

\subsection{Constitutive relations}

Guided by (13), we identify $\varphi, \operatorname{grad} \varphi$, and $\boldsymbol{\jmath}$ as independent constitutive variables and $\psi, \mu-\pi, \xi$, and $\zeta$ as dependent constitutive variables. As consequences of ensuring that the reduced free-energy imbalance (4) holds in all processes, we then find that:

- $\psi$ must be independent of $\boldsymbol{\jmath}$ and, thus, be given by a relation of the form

$$
\psi=\hat{\psi}(\varphi, \operatorname{grad} \varphi)
$$

- $\mu-\pi$ and $\xi$ must be generated from derivatives of the response function $\hat{\psi}$ through the relations

$$
\left.\begin{array}{rl}
\mu-\pi & =\frac{\partial \hat{\psi}(\varphi, \operatorname{grad} \varphi)}{\partial \varphi}, \\
\xi & =\frac{\partial \hat{\psi}(\varphi, \operatorname{grad} \varphi)}{\partial(\operatorname{grad} \varphi)},
\end{array}\right\}
$$

and, thus, must also be independent of $\boldsymbol{j}$;

- the response function $\hat{\zeta}$ determining $\zeta$ must satisfy the residual free-energy imbalance

$$
\hat{\zeta}(\varphi, \operatorname{grad} \varphi, \boldsymbol{\jmath}) \cdot \boldsymbol{\jmath} \leq 0 .
$$

Noticing from (4), (9), and (12) 1 that the pointwise microforce balance (4) admits the equivalent form $\mu=(\mu-\pi)-\operatorname{div} \xi-\gamma$

and invoking the constitutive relations $(15)_{1}$ and (15) for $\mu-\pi$ and $\xi$, we find that the constitutively 
augmented pointwise microforce balance determines the chemical potential in the form

$$
\mu=\frac{\partial \hat{\psi}(\varphi, \operatorname{grad} \varphi)}{\partial \varphi}-\operatorname{div}\left(\frac{\partial \hat{\psi}(\varphi, \operatorname{grad} \varphi)}{\partial(\operatorname{grad} \varphi)}\right)-\gamma .
$$

The following remarks are in order:

- The pointwise free-energy imbalance (13) involves the active parts $\mu_{a}$ and $\pi_{a}$ of the chemical potential $\mu$ and the internal microforce $\pi$ only through the difference $\mu_{a}-\pi_{a}=\mu-\pi$. Thus, whereas $(15)_{2}$ yields a constitutive relation for that difference, the components $\mu_{a}$ and $\pi_{a}$ of that difference remain individually unspecified. We nevertheless arrive at a complete specification of the chemical potential $\mu=\mu_{a}+\mu_{r}$, as (18) shows. Specifically, the chemical potential is determined by the microforce balance.

- Although $\mu_{a}$ and $\pi_{a}$ need not be specified independently to arrive at (18), doing so impacts the interpretation of certain salient quantities. For instance, the legitimate choice $\mu_{a} \equiv 0$ implies that the chemical potential $\mu$ is a reaction that enters the theory to ensure that the internal constraint (8) is met and a completely analogous statement applies regarding the role of the internal microforce $\pi$ for the complementary choice $\pi_{a} \equiv 0$. The freedom to select $\mu_{a}$ and $\pi_{a}$ independently in any manner consistent with (15) should not be overlooked, as certain particular choices might be advantageous.

- According to (18), the chemical potential $\mu$ splits into two contributions, one involving the free energy density $\psi$ and other involving the external microforce density $\gamma$. Hence, these contributions can be called internal and external parts of the chemical potential, respectively. The latter quantity can represent, for instance, the gravitational potential energy or the electric potential. Therefore, the introduction of the microforce balance (3) and the internal constraint (8) might be advantageous even in the standard treatment of diffusion. A more general perspective of this nature underpins a theory for constituent diffusion proposed by Fried and Sellers [5, 14], in which the constituent flux $\boldsymbol{J}$ is viewed as an additional kinematical descriptor and an additional microforce balance is introduced.

\subsection{Summary of the evolution equations}

Augmenting the pointwise constituent content and microforce balances (2) and (4) with the thermodynamically compatible constitutive relations $(15)_{1}$ and $(15)_{2}$ for $\mu_{a}-\pi_{a}$ and $\xi$, we arrive at the system of equations

$$
\left.\begin{array}{rl}
\dot{\varphi}= & \operatorname{div} \hat{\boldsymbol{\jmath}}(\varphi, \operatorname{grad} \mu)+m, \\
\mu= & \frac{\partial \hat{\psi}(\varphi, \operatorname{grad} \varphi)}{\partial \varphi} \\
& \quad-\operatorname{div}\left(\frac{\partial \hat{\psi}(\varphi, \operatorname{grad} \varphi)}{\partial(\operatorname{grad} \varphi)}\right)-\gamma .
\end{array}\right\}
$$

In writing (19), we have assumed that the relation $\operatorname{grad} \mu=\hat{\zeta}(\varphi, \operatorname{grad} \varphi, \boldsymbol{\jmath})$ (with the response function $\hat{\zeta}$ satisfying the residual free-energy imbalance (16)) can be inverted to give

$\boldsymbol{\jmath}=\hat{\boldsymbol{\jmath}}(\varphi, \operatorname{grad} \mu)$.

Recalling that $\mu_{a} \equiv 0$ implies that the chemical potential $\mu$ is purely a reaction that enters the theory to ensure that the internal constraint $c=\varphi$ is maintained, (19) is to be solved for the phase field $\varphi$ and the reaction $\mu$. This system should be supplemented by an initial condition for $\varphi$ and a pair of boundary conditions. We postpone the discussion of boundary conditions to Sect. 5.

\subsection{Specialized theory}

We consider a simple theory in which, sufficient to ensure that (16) holds, $\hat{\zeta}$ is independent of $\operatorname{grad} \varphi$ and of the particular form

$\hat{\zeta}(\varphi, \operatorname{grad} \varphi, \boldsymbol{\jmath})=-\boldsymbol{K}^{-1}(\varphi) \boldsymbol{\jmath}$,

where the mobility $\boldsymbol{K}$ is assumed to be positive definite for all values of its argument. With the choice (21) and recalling from (7) that $\zeta=\operatorname{grad} \mu$, we find from (18) that the constituent flux is given by 


$$
\begin{aligned}
\boldsymbol{J}= & -\boldsymbol{K}(\varphi) \operatorname{grad}\left(\frac{\partial \hat{\psi}(\varphi, \operatorname{grad} \varphi)}{\partial \varphi}\right. \\
& \left.-\operatorname{div}\left(\frac{\partial \hat{\psi}(\varphi, \operatorname{grad} \varphi)}{\partial(\operatorname{grad} \varphi)}\right)-\gamma\right) .
\end{aligned}
$$

Next, bearing in mind the constraint (8), we may use (22) in the pointwise constituent content balance (2) to yield a single evolution equation,

$$
\begin{aligned}
\dot{\varphi}= & \operatorname{div}\left(\boldsymbol { K } ( \varphi ) \operatorname { g r a d } \left(\frac{\partial \hat{\psi}(\varphi, \operatorname{grad} \varphi)}{\partial \varphi}\right.\right. \\
& \left.\left.-\operatorname{div}\left(\frac{\partial \hat{\psi}(\varphi, \operatorname{grad} \varphi)}{\partial(\operatorname{grad} \varphi)}\right)-\gamma\right)\right)+m,
\end{aligned}
$$

for the phase field $\varphi$. If we next replace $\varphi$ by $\rho$, then (23) is identical to (3.17) of Gurtin [3].

Finally, granted that the response function $\hat{\psi}$ determining the free-energy density $\psi$ has the particular form

$$
\hat{\psi}(\varphi, \operatorname{grad} \varphi)=f(\varphi)+\frac{\lambda}{2}|\operatorname{grad} \varphi|^{2},
$$

with $f$ a double-well potential and $\lambda>0$ a constant gradient-energy modulus, that the mobility is independent of the phase field and isotropic, whereby

$$
\boldsymbol{K}(\varphi)=\kappa \boldsymbol{I},
$$

with $\kappa>0$ being a constant scalar mobility, and that the external microforce density $\gamma$ and external supply rate $m$ of constituent content vanish, (23) reduces to the classical Cahn-Hilliard equation

$$
\dot{\varphi}=\kappa \Delta\left(f^{\prime}(\varphi)-\lambda \triangle \varphi\right) .
$$

Notice that (26) can be written equivalently as a system

$$
\left.\begin{array}{l}
\dot{\varphi}=\kappa \Delta \mu, \\
\mu=f^{\prime}(\varphi)-\lambda \Delta \varphi,
\end{array}\right\}
$$

which must be solved for the fields $\varphi$ and $\mu$.

Suppose that there is reason for determining the reactive contribution $\mu_{r}$ to the chemical potential. This can be achieved by specifying $\mu_{a}$ and rewriting (27) in terms of $\mu_{a}$ and $\mu_{r}$. For the particular choice $\mu_{a} \equiv 0$, we have $\mu=\mu_{r}$ but (27) is unchanged. If, however, we insist also that $\mu_{a}=f^{\prime}(\varphi)$, we then arrive at an alternative system for $\varphi$ and $\mu_{r}$ of the form

$$
\left.\begin{array}{rl}
\dot{\varphi} & =\kappa \Delta\left(\mu_{r}+f^{\prime}(\varphi)\right), \\
\mu_{r} & =-\lambda \Delta \varphi .
\end{array}\right\}
$$

\section{Allen-Cahn type theory coupled with diffusion}

In this section, we derive a general theory of the Allen-Cahn type coupled with constituent transport. Towards this end, we find that it suffices to augment the constituent-content and microforce balances (2) and (4) with a certain class of constitutive relations.

\subsection{Constitutive relations}

Guided by (6), we identify $c, \varphi, \operatorname{grad} \varphi, \dot{\varphi}$, and $\boldsymbol{\jmath}$ as independent constitutive variables and $\psi, \mu, \pi, \xi$, and $\zeta$ as dependent constitutive variables. Further, we decompose $\pi$ as

$\pi=\pi_{e}+\pi_{d}$,

where $\pi_{e}$ and $\pi_{d}$ are defined such that

$\pi_{e}=\pi(c, \varphi, \operatorname{grad} \varphi, 0, \mathbf{0})$

and

$\pi_{d}=\pi(c, \varphi, \operatorname{grad} \varphi, \dot{\varphi}, \boldsymbol{\jmath})-\pi_{e}$.

With these provisions, we find that: $\psi$ must be independent of $\boldsymbol{J}$ and, thus, be given by a relation of the form

$\psi=\tilde{\psi}(c, \varphi, \operatorname{grad} \varphi)$

$\mu, \xi$, and $\pi_{e}$ must be generated from derivatives of the response function $\tilde{\psi}$ through the relations

$$
\left.\begin{array}{c}
\mu=\frac{\partial \tilde{\psi}(c, \varphi, \operatorname{grad} \varphi)}{\partial c}, \\
\xi=\frac{\partial \tilde{\psi}(c, \varphi, \operatorname{grad} \varphi)}{\partial(\operatorname{grad} \varphi)}, \\
\pi_{e}=-\frac{\partial \tilde{\psi}(c, \varphi, \operatorname{grad} \varphi)}{\partial \varphi},
\end{array}\right\}
$$

and, thus, must also be independent of $\boldsymbol{j}$; the response functions $\pi_{d}$ and $\hat{\zeta}$ determining $\pi-\pi_{e}$ and $\zeta$ must satisfy the residual free-energy imbalance 


$$
\begin{aligned}
& \pi_{d}(c, \varphi, \operatorname{grad} \varphi, \dot{\varphi}, \boldsymbol{\jmath}) \dot{\varphi} \\
& \quad+\hat{\zeta}(c, \varphi, \operatorname{grad} \varphi, \dot{\varphi}, \boldsymbol{\jmath}) \cdot \boldsymbol{\jmath} \leq 0 .
\end{aligned}
$$

Instead of considering the most general expressions $\pi_{d}$ and $\hat{\zeta}$ satisfying (34), we content ourselves with the choices

$$
\left.\begin{array}{c}
\pi_{d}(c, \varphi, \operatorname{grad} \varphi, \dot{\varphi}, \boldsymbol{\jmath})=-\beta(\varphi, \dot{\varphi}) \dot{\varphi}, \\
\hat{\boldsymbol{\zeta}}(c, \varphi, \operatorname{grad} \varphi, \dot{\varphi}, \boldsymbol{\jmath})=-\boldsymbol{K}^{-1}(c) \boldsymbol{\jmath},
\end{array}\right\}
$$

where the kinetic modulus $\beta$ is nonnegative for each choice of its arguments and, as previously, the mobility $\boldsymbol{K}$ is positive definite for each choice of its argument.

\subsection{Governing equations}

The governing equations of the theory are obtained by augmenting the pointwise constituent content balance (2) and the pointwise microforce balance (4) with the thermodynamically compatible constitutive relations (33) and (35). The resulting system of equations is comprised by an evolution equation

$$
\dot{c}=\operatorname{div}\left(\boldsymbol{K}(c) \operatorname{grad}\left(\frac{\partial \tilde{\psi}(c, \varphi, \operatorname{grad} \varphi)}{\partial c}\right)\right)+m
$$

for the mass fraction $c$ coupled to an evolution equation

$$
\begin{aligned}
\beta(\varphi, \dot{\varphi}) \dot{\varphi}= & \operatorname{div}\left(\frac{\partial \tilde{\psi}(c, \varphi, \operatorname{grad} \varphi)}{\partial(\operatorname{grad} \varphi)}\right) \\
& -\frac{\partial \tilde{\psi}(c, \varphi, \operatorname{grad} \varphi)}{\partial \varphi}+\gamma
\end{aligned}
$$

for the phase field $\varphi$. This system requires initial conditions for both $c$ and $\varphi$. The matter of boundary conditions is considered in Sect. 5.

\subsection{Specialized theory}

Our next aim is to introduce a simple theory that can serve to approximate the Cahn-Hilliard eq. (26). The theory is based on:

- Choosing the constitutive response function $\tilde{\psi}$ determining the free-energy density $\psi$ such that

$$
\tilde{\psi}(c, \varphi, \operatorname{grad} \varphi)=f(\varphi)+\frac{\lambda}{2}|\operatorname{grad} \varphi|^{2}+\frac{\chi}{2}(c-\varphi)^{2},
$$

with $f$ and $\lambda$ being identical to the corresponding quantities in (24) and with $\chi>0$ being a constant coupling energy modulus, in which case $(33)_{2-4}$ specialize to

$$
\left.\begin{array}{rl}
\mu & =\chi(c-\varphi), \\
\xi & =\lambda \operatorname{grad} \varphi, \\
\pi_{e} & =-f^{\prime}(\varphi)-\chi(\varphi-c) .
\end{array}\right\}
$$

- Choosing the kinetic modulus and mobility such that

$\beta=0 \quad$ and $\quad \boldsymbol{K}=\kappa \boldsymbol{1}$,

with $\kappa>0$ being identical to the corresponding quantity appearing in (25).

With (38)-(40) and the assumption that the external microforce density $\gamma$ and external supply rate $m$ of constituent content vanish, the system (36), (37) simplifies to

$$
\left.\begin{array}{l}
\dot{c}=\kappa \chi \Delta(c-\varphi), \\
0=\lambda \Delta \varphi-f^{\prime}(\varphi)-\chi(\varphi-c) .
\end{array}\right\}
$$

The system (41) can be written in various equivalent ways. From the particular alternative

$$
\left.\begin{array}{l}
\dot{c}=\kappa \Delta\left(f^{\prime}(\varphi)-\lambda \Delta \varphi\right), \\
c=\varphi+\frac{f^{\prime}(\varphi)-\lambda \Delta \varphi}{\chi},
\end{array}\right\}
$$

we are led to conjecture that the Cahn-Hilliard equation (26) should be recovered in the limit as $\chi$ tends to infinity. Furthermore, on comparing $(27)_{2}$ to $(41)_{2}$, we hypothesize that $\chi(\varphi-c)$ should tend to the chemical potential $\mu$ of the Cahn-Hilliard theory as $\chi$ tends to infinity. Another alternative to (41) arises on using (39) $)_{1}$ to eliminate the constituent concentration $c$ in favor of the chemical potential $\mu$, leading to the system

$$
\left.\begin{array}{l}
\dot{\varphi}=\kappa \Delta \mu-\frac{\dot{\mu}}{\chi}, \\
\mu=f^{\prime}(\varphi)-\lambda \Delta \varphi .
\end{array}\right\}
$$


The systems of equations described above must be supplemented with appropriate initial and boundary conditions. Whereas (41) and (42) require initial condition for the constituent concentration $c$, (43) requires an initial condition for $\varphi+\mu / \chi$. The issue of boundary conditions will be addressed next.

\section{Boundary conditions}

Following the procedure advanced in a thermomechanical context by Fried and Gurtin [15], we now consider the localization of the basic laws for points at the boundary $\partial \mathcal{B}$ of $\mathcal{B}$ with the objective of formulating boundary conditions for the theories developed in Sects. 3 and 4 . We begin by formulating the basic laws for boundary pillboxes which include infinitesimal portions of the environment and the body, assuming throughout that $\partial \mathcal{B}$ is a structureless surface within which constituent transport is negligible. We emphasize that this treatment is completely independent of the constitutive theories developed in Sects. 3.2 and 4.1.

For a boundary pillbox $\mathcal{A}$, the constituent content and microforce balances require that

$$
\int_{\mathcal{A}}\left(\jmath_{\mathrm{env}}+\boldsymbol{\jmath} \cdot \boldsymbol{n}\right) \mathrm{d} a=0
$$

and

$$
\int_{\mathcal{A}}\left(\xi_{\text {env }}-\boldsymbol{\xi} \cdot \boldsymbol{n}\right) \mathrm{d} a=0,
$$

where $\boldsymbol{n}$ denotes the unit normal to $\partial \mathcal{B}$, directed outward from $\mathcal{B}$, Jenv and $\xi_{\text {env }}$ represent, respectively, the constituent flow into $\mathcal{A}$ from the environment and the microtraction exerted on $\mathcal{A}$ by the environment, and $\boldsymbol{J} \cdot \boldsymbol{n}$ and $-\boldsymbol{\xi} \cdot \boldsymbol{n}$ represent, respectively, the constituent flow into $\mathcal{A}$ from $\mathcal{B}$ and the microtraction exerted on $\mathcal{A}$ by the material in $\mathcal{B}$. Since $\mathcal{A}$ is arbitrary, (44), (45) localize to yield pointwise conditions

$$
\jmath_{\mathrm{env}}+\boldsymbol{\jmath} \cdot \boldsymbol{n}=0
$$

and

$$
\xi_{\mathrm{env}}-\boldsymbol{\xi} \cdot \boldsymbol{n}=0
$$

on $\partial \mathcal{B}$. Additionally, the free-energy imbalance for $\mathcal{A}$ requires that
$\int_{\mathcal{A}}\left(\jmath_{\mathrm{env}} \mu_{\mathrm{env}}+\xi_{\mathrm{env}} \dot{\varphi}_{\mathrm{env}}+(\mu \boldsymbol{\jmath}-\dot{\varphi} \boldsymbol{\xi}) \cdot \boldsymbol{n}\right) \mathrm{d} a \geq 0$,

where $\mu_{\mathrm{env}}$ and $\varphi_{\mathrm{env}}$ are the limits of the environmental chemical potential and the phase field at $\partial \mathcal{B}$. Whereas the first two integral contributions of (48) represents the energy inflow into $\mathcal{A}$ from the environment, the remaining two represent the energy inflow into $\mathcal{A}$ from $\mathcal{B}$. On taking (46), (47) into account, it thus follows from (48) and the arbitrariness of $\mathcal{A}$ that

$\jmath_{\mathrm{env}}\left(\mu_{\mathrm{env}}-\mu\right)+\xi_{\mathrm{env}}\left(\dot{\varphi}_{\mathrm{env}}-\dot{\varphi}\right) \geq 0$.

The left-hand side of the pointwise inequality (49) represents the interfacial dissipation. To ensure that (49) holds, it is sufficient but certainly not necessary to require that

$\jmath_{\mathrm{env}}\left(\mu_{\mathrm{env}}-\mu\right)=0 \quad$ and $\quad \xi_{\mathrm{env}}\left(\dot{\varphi}_{\mathrm{env}}-\dot{\varphi}\right)=0$.

Fried and Gurtin [15] refer to stipulations of the form (50) as "uncoupled zero-dissipation conditions".

Although (46), (47) and (49) can be used to formulate boundary conditions that allow coupling between constituent transport and microstructural evolution while accounting for dissipative interactions with the environment, we work instead with (46), (47) and the conditions (50) sufficient to satisfy (49). It is evident that the following classes of boundary conditions, posed for an arbitrary subsurface $\mathcal{S}$ of $\partial \mathcal{B}$, are consistent with (46), (47) and (50):

- Assigned constituent flow across $\mathcal{S}$ : For this class of boundary conditions, $\jmath_{\mathrm{env}}$ is given and $\boldsymbol{J} \cdot \boldsymbol{n}$ is prescribed on $\mathcal{S}$ in accord with

$\boldsymbol{\jmath} \cdot \boldsymbol{n}=-\jmath_{\mathrm{env}}$,

(46) is trivially satisfied on $\mathcal{S}, \mu_{\text {env }}$ is determined on $\mathcal{S}$ through (50) $)_{1}$ granted that $\jmath_{\text {env }} \neq 0$. If $\jmath_{\text {env }}=0$, then $\mathcal{B}$ is chemically insulated from the environment on the subset of $\mathcal{S}$ of $\partial \mathcal{B}$ and (50) 1 is trivially satisfied.

- Assigned chemical potential on $\mathcal{S}$ : For this class of boundary conditions, $\mu_{\text {env }}$ is given and $\mu$ is prescribed on $\mathcal{S}$ in accord with

$\mu=\mu_{\mathrm{env}}$,

$\jmath_{\text {env }}$ is determined on $\mathcal{S}$ through (46), and (50) 1 is trivially satisfied on $\mathcal{S}$. If, in particular, $\mu_{\text {env }}=$ constant, then $\mathcal{B}$ is in contact with a 
reservoir of uniform chemical potential on the subset of $\mathcal{S}$ of $\partial \mathcal{B}$.

- Assigned microtraction on $\mathcal{S}$ : For this class of boundary conditions, $\xi_{\text {env }}$ is given and $\boldsymbol{\xi} \cdot \boldsymbol{n}$ is prescribed on $\mathcal{S}$ in accord with

$$
\boldsymbol{\xi} \cdot \boldsymbol{n}=\xi_{\mathrm{env}},
$$

(47) is trivially satisfied on $\mathcal{S}$, and $\dot{\varphi}_{\text {env }}$ is determined on $\mathcal{S}$ through $(50)_{2}$ granted that $\xi_{\text {env }} \neq 0$. If $\xi_{\text {env }}=0$, then $\mathcal{B}$ is free of microtraction and $(50)_{2}$ is trivially satisfied.

- Assigned microstructure on $\mathcal{S}$ : For this class of boundary conditions, $\varphi_{\text {env }}$ is given and $\varphi$ is assigned on $\mathcal{S}$ in accord with

$$
\varphi=\varphi_{\mathrm{env}},
$$

$\xi_{\text {env }}$ is determined on $\mathcal{S}$ by (47), and $(50)_{2}$ is trivially satisfied on $\mathcal{S}$.

The boundary conditions (51) and (52) are commonly encountered as mutually exclusive alternatives in conventional formulations of problems involving constituent transport, which allow one boundary condition on any open subset of the boundary. Therefore, (51) and (52) cannot be used on a common portion of $\partial \mathcal{B}$ at the same time. The boundary condition (53) is commonly invoked in the context of microforce-based phase field problems, which also require just one boundary condition on any open subset of $\partial \mathcal{B}$. Although the remaining boundary condition (54) is a mathematically viable alternative to (53), its use is difficult to justify from a physical perspective.

The foregoing discussion of boundary conditions was based exclusively on the local forms of the basic laws on the interface between the body and its surrounding environment, namely the constituent content balance (46), the microforce balance (47), and the free-energy imbalance (49). Therefore, it applies to both classes of diffusion theories considered here. However, the following remarks are in order:

- The boundary conditions (51) and (52) are conditions on the normal component of the constituent flux and chemical potential and, hence, have the same physical interpretation for the two classes of diffusion theories considered here. On the other hand, the interpretation of the boundary conditions (53) and (54) depends on the meaning of the phase field itself. Consider, for instance, the specialized theories introduced in Subsections 3.4 and 4.3, which respectively lead to the Cahn-Hilliard theory and its Allen-Cahn type regularization. In both cases, (53) and (54) turn out to be boundary conditions on the normal derivative of the phase field and the value of the phase field. For the CahnHilliard theory, since the concentration and phase field coincide, (53) and (54) yield boundary conditions for the concentration and its the normal derivative, whereas for the Allen-Cahn type theory (53) and (54) are boundary conditions on the normal derivative and value of a combination of the concentration and chemical potential, as is evident from (39).

- The boundary conditions for the Cahn-Hilliard theory that are commonly adopted in the literature require that the normal derivative of both the constituent chemical potential and the concentration vanish at the boundary. In view of (39), these conditions are equivalent to homogeneous Neumann boundary conditions for the constituent chemical potential and the phase field in the Allen-Cahn type diffusion theory.

- The issue of boundary conditions for the CahnHilliard equation has been addressed in other works specially to generalize the standard boundary conditions to incorporate short-ranged interactions between the mixture and the walls in confined systems. These generalizations give rise to what have been referred to as dynamic boundary conditions. Researchers who have considered such conditions include Binder and Frisch [16], Fischer et al. [17], Kenzler et al. [18], Goldstein et al. [19], Heida [20], Liu and Wu [21], Fukao [22], and Colli and Fukao [23]. In this case, the body-environment interface cannot be treated as structureless anymore.

\section{Numerical results}

To explore the viability of approximating solutions of the Cahn-Hilliard equation (26) by solutions of the Allen-Cahn type diffusion system (43), we consider the three-dimensional version of the benchmark problem for the Cahn-Hilliard equation formulated and studied by Jeong et al. [24]. 
For Jeong et al. [24], the phase-field variable represents the difference of the concentration of the two components of a binary mixture and thus should, in principle, be confined to the interval $[-1,1]$. This expectation is not, however, met by the numerical solutions to the benchmark problem. This is a recognized drawback of the classical version of the Cahn-Hilliard equation, which is predicated on assuming that the mobility is constant and that the homogeneous contribution to the free-energy density is given by a symmetric double-well potential with wells at \pm 1 that does not strongly penalize values of the phase field outside of $[-1,1]$. Detailed discussions of this issue are provided by Elliott and Garcke [25] and Novick-Cohen [26], among others.

\subsection{Dimensionless evolution equations}

To formulate initial-boundary-value problems consistent with that considered by Jeong et al. [24], we introduce characteristic measures $L$ and $E$ of length and energy and we identify the ratio

$$
\frac{L^{5}}{E \kappa}=T
$$

as a characteristic measure of time. In addition, we choose the double-well potential $f$ to be of the particular form

$f(\varphi)=\frac{E}{L^{3}} \varphi^{2}(1-\varphi)^{2}$

and, noticing that the gradient energy modulus $\lambda$ and the coupling energy modulus $\chi$ carry dimensions of energy per unit length and energy per unit volume, respectively, define the dimensionless gradient energy modulus $\sigma>0$ and the dimensionless coupling energy coefficient $l>0$ by

$\sigma=\frac{L \lambda}{E} \quad$ and $\quad l=\frac{E}{L^{3} \chi}$.

Adopting a scaling in which lengths, times, and energies are measured relative to $L, T$, and $E$, respectively, and referring to (56) and (57), we thus find that the dimensionless version of the free-energy density (24) underlying the Cahn-Hilliard equation (26) is given by
$\psi_{\mathrm{CH}}=\frac{\hat{\psi}(\varphi, \operatorname{grad} \varphi)}{E / L^{3}}=\varphi^{2}(1-\varphi)^{2}+\frac{\sigma}{2}|\operatorname{grad} \varphi|^{2}$,

where, in an abuse of notation, the symbol 'grad' used previously to denote the gradient now (and hereafter) denotes the dimensionless gradient. Moreover, we find that the dimensionless version of the evolution equation (26) has the form

$\dot{\varphi}=\Delta(2 \varphi(1-\varphi)(1-2 \varphi)-\sigma \Delta \varphi)$,

where, in a further abuse of notation, a superposed dot and a triangle now (and hereafter) denote the partial derivative with respect to dimensionless time and the dimensionless Laplacian.

Analogously, we find that the dimensionless version of the free-energy density (38) for the AllenCahn type diffusion theory is given by

$$
\begin{aligned}
\psi_{\mathrm{AC}} & =\frac{\tilde{\psi}(c, \varphi, \operatorname{grad} \varphi)}{E / L^{3}} \\
& =\varphi^{2}(1-\varphi)^{2}+\frac{\sigma}{2}|\operatorname{grad} \varphi|^{2}+\frac{1}{2 l}(c-\varphi)^{2}
\end{aligned}
$$

and, on introducing the dimensionless chemical potential

$v=\frac{\mu}{E / L^{3}}=\frac{c-\varphi}{l}$,

we see that the dimensionless version of the associated system (43) for the Allen-Cahn type diffusion theory has the form

$$
\left.\begin{array}{rl}
\dot{\varphi} & =\Delta v-i \dot{v}, \\
v & =2 \varphi(1-\varphi)(1-2 \varphi)-\sigma \Delta \varphi .
\end{array}\right\}
$$

For later use, we see from (61) that, in the context of the Allen-Cahn type diffusion theory, the constituent concentration is given by

$c=\varphi+\imath$.

Moreover, we use (61) and (62) 2 to eliminate $c$ from (60) and thereby obtain an alternative representation,

$\psi_{\mathrm{AC}}=\varphi^{2}(1-\varphi)^{2}+\frac{\sigma}{2}|\operatorname{grad} \varphi|^{2}+\frac{l}{2} v^{2}$,

for the dimensionless free-energy density of the Allen-Cahn type diffusion theory. Although the representation (64) merely provides a means to evaluate $\psi_{\mathrm{AC}}$ in terms of $\varphi, \operatorname{grad} \varphi$, and the 
dimensionless chemical potential $v$, it should not be misinterpreted as a constitutive relation.

\subsection{Boundary and initial conditions}

Consistent with the work of Jeong et al. [24], we consider the dimensionless Cahn-Hilliard equation (59) subject to the natural boundary conditions

$\operatorname{grad} \Delta \varphi \cdot v=0, \quad \operatorname{grad} \varphi \cdot v=0, \quad$ on $\quad \partial \mathcal{R}$,

where $v$ is the outward unit normal at all smooth points of the boundary $\partial \mathcal{R}$ of the dimensionless cube $\mathcal{R}$. Since, by $(62)_{2}$ and (65),

$\operatorname{grad} v=2\left(1-6 \varphi+6 \varphi^{2}\right) \operatorname{grad} \varphi-\sigma \operatorname{grad} \Delta \varphi$,

we infer that the natural boundary conditions

$\operatorname{grad} v \cdot \boldsymbol{v}=0, \quad \operatorname{grad} \varphi \cdot \boldsymbol{v}=0, \quad$ on $\quad \partial \mathcal{R}$,

for the dimensionless Allen-Cahn type diffusion system (62) are equivalent to (65). Granted our choice of scales, (65) and (67) are the dimensionless versions of the boundary conditions (51) and (53) for $\jmath_{\mathrm{env}}=0$ and $\xi_{\text {env }}=0$ that correspond, respectively, to (65) and (67). These conditions require that the normal derivatives of the constituent chemical potential and concentration vanish on $\partial \mathcal{R}$ for both diffusion theories considered here. For the Cahn-Hilliard diffusion theory, this requirement is immediate since $\varphi$ coincides with the constituent concentration $c$. For the Allen-Cahn type diffusion theory, it hinges on the specific form (61) of the dimensionless chemical potential and $(67)_{2}$.

The initial condition for the dimensionless CahnHilliard equation (59) is simply

$\varphi(\cdot, 0)=c_{0}, \quad$ on $\quad \mathcal{R}$,

where $c_{0}$ is the prescribed constituent concentration at dimensionless time $t=0$. With reference to (63), the corresponding initial condition for the Allen-Cahn type diffusion system (62) is

$\varphi(\cdot, 0)+v v(\cdot, 0)=c_{0}, \quad$ on $\quad \mathcal{R}$.
6.3 Weak versions of the evolution equations for natural boundary conditions

We work with weak versions of the dimensionless Cahn-Hilliard eq. (59) and the dimensionless AllenCahn type diffusion system (62) subject to the respective natural boundary conditions (65) and (67).

For (59) and (65), we multiply (59) by a smooth test field $w$ satisfying $\operatorname{grad} w \cdot \boldsymbol{v}=0$ on $\partial \mathcal{R}$, integrate the resulting identity over $\mathcal{R}$, apply the divergence theorem, and invoke (65), giving

$$
\begin{aligned}
\int_{\mathcal{R}} w \dot{\varphi} \mathrm{d} v= & \sigma \int_{\mathcal{R}} \Delta w \Delta \varphi \mathrm{d} v \\
& -2 \int_{\mathcal{R}} \varphi(1-\varphi)(1-2 \varphi) \operatorname{grad} w \cdot \operatorname{grad} \varphi \mathrm{d} v
\end{aligned}
$$

Similarly, for (62) and (67), we multiply $(62)_{1}$ by a smooth test field $w$ and $(62)_{2}$ by a smooth test field $q$, integrate both of the resulting identities over $\mathcal{R}$, apply the divergence theorem, and invoke (67), giving

$$
\left.\begin{array}{rl}
\int_{\mathcal{R}} w \dot{\varphi} \mathrm{d} v= & -\int_{\mathcal{R}} \operatorname{grad} w \cdot \operatorname{grad} v \mathrm{~d} v \\
& -\imath \int_{\mathcal{R}} w \dot{\mathrm{d}} \mathrm{d} v \\
\int_{\mathcal{R}} q v \mathrm{~d} v= & 2 \int_{\mathcal{R}} q \varphi(1-\varphi)(1-2 \varphi) \mathrm{d} v \\
& +\sigma \int_{\mathcal{R}} \operatorname{grad} q \cdot \operatorname{grad} \varphi \mathrm{d} v .
\end{array}\right\}
$$

\subsection{Spatial and temporal discretizations}

We divide the region $\mathcal{R}$ into a uniform mesh of $128^{3}$ cubes and use isogeometric finite elements of the type developed by Hughes et al. [27] to discretize (70) over that mesh. Since the Laplacians of the test field $w$ and the trial solution $\varphi$ appear in (70), we employ test fields and trial solutions of polynomial order three and interelemental continuity two, as the spatial resolution afforded by this choice is sufficient to yield the accuracy and resolution needed to provide an accurate baseline for exploring the fidelity of the solutions arising from the Allen-Cahn type diffusion theory. Working on the same uniform mesh of $128^{3}$ cubes and recognizing that only the first gradients of the test fields $w$ and $q$ and the trial solution $\varphi$ appear in (62), 
we also discretize (62) with isogeometric finite elements but, in so doing, employ test fields and trial solutions of polynomial order two and interelemental continuity unity.

To discretize (70) and (71) with respect to the dimensionless time $t$, we apply the second-order accurate generalized- $\alpha$ method of Chung and Hulbert [28] with uniform step size $10^{-4}$. That method requires the provision of a spectral radius which is associated with numerical dissipation and can take values between zero and unity, inclusive. If the spectral radius is equal to unity, no frequencies are damped; if the spectral radius is close to unity, a corresponding range of high frequencies is damped; if the spectral radius is equal to zero, all frequencies are damped. Based on prior work of Sarmiento et al. [29], we set the value of the spectral radius equal to 0.9 .

Our choices of spatial and temporal discretization schemes for (70) have been applied previously by Gómez et al. [30] and Vignal et al. [31] to construct numerical approximations to solutions of various initial-boundary-value problems for the Cahn-Hilliard equation. These choices for the discretization schemes were dictated by convenience only and hence any comparison to alternative schemes is outside the scope of this paper.

\subsection{Comparisons}

To enable comparisons with the simulations of Jeong et al. [24], we choose the value

$$
\sigma=\frac{1}{512 \operatorname{arctanh}^{2}(9 / 10)}
$$

for the dimensionless gradient energy modulus and take the dimensionless open cube $\mathcal{R}$ to be of side length 2. Recalling that our discretization rests on the uniform division of $\mathcal{R}$ into $128^{3}$ cubes, we furthermore choose the value of the initial constituent concentration $c_{0}$ to be given by

$$
c_{0}(\boldsymbol{x})=\frac{1}{2}\left(\tanh \left(\frac{1}{2 \sqrt{\sigma}}\left(\frac{1}{10}-d(\boldsymbol{x})\right)\right)+1\right),
$$

with

$$
d(\boldsymbol{x})=\left(x_{1}^{2}+\left(x_{2}-\frac{1}{128}\right)^{2}+\left(x_{3}-\frac{1}{128}\right)^{2}-\frac{3}{4}\right)^{1 / 2},
$$

where $x_{i}=(\boldsymbol{x}-\boldsymbol{o}) \cdot \boldsymbol{e}_{i}$ satisfying $\left|x_{i}\right| \leq 1, i=1,2,3$, denote the components of $\boldsymbol{x}$ relative to a fixed orthonormal basis $\left\{\boldsymbol{e}_{1}, \boldsymbol{e}_{2}, \boldsymbol{e}_{3}\right\}$ and the origin $\boldsymbol{o}$ lies at the center of the cube $\mathcal{R}$.

We use the spatial and temporal discretization schemes described in the previous subsection to construct numerical solutions to the initial-value problems for (70) and (71), bearing in mind that the respective initial conditions for those problems are determined by using the particular expression (73) for the initial constituent concentration $c_{0}$ in (68) and (69). Although the initial-value problem (70) for the CahnHilliard theory is completely determined without additional input, its counterpart, (71), for the AllenCahn type diffusion theory requires input in the form of the dimensionless coupling energy coefficient $l$. The solution to the initial value problem for (70) serves as a reference with respect to which we compare solutions to the initial-value problem for (71) for various values of $l$. To test our previously stated conjecture that the Cahn-Hilliard equation (26) should be recovered from the Allen-Cahn type diffusion system in the limit as $\chi \rightarrow \infty$ or, equivalently, by $(57)_{2}$, as $\imath \rightarrow 0$, we consider values of $l$ ranging from $l=8.0 \times 10^{0}$ to $l=4.0 \times 10^{-2}$. Our simulations are conducted up to dimensionless time $t=1.5$ and thus include the interval considered by Jeong et al. [24, Table 4], who terminate their simulations at dimensionless time $t \approx 1.2$.

\subsubsection{Cahn-Hilliard theory}

The initial distribution of $\varphi$ determined by (68) and (72)-(73) involves a spherical shell within which $\varphi=1$. That shell is bounded by transition layers, also spherical shells, within which $0<\varphi<1$ and outside of which $\varphi=0$. As the dimensionless time $t$ increases from $t_{i}=0.0$ to $t_{f}=1.5$, the transition layers remain roughly concentric and spherical and propagate toward the origin, the inner one at a velocity exceeding that of the outer one. At a critical time $t^{*} \approx 1.283$, the inclusion where $\varphi=0$ collapses and the inner transition layer disappears, leaving a transition layer between a roughly spherical inclusion within which $\varphi \approx 1$ and the remainder of $\mathcal{R}$, within which $\varphi \approx 0$. The system then equilibrates by what appear to be small adjustments to the distribution of $\varphi$. The 
observed sequence of events reflects the decay of the dimensionless total free-energy

$$
\Psi_{\text {Сн }}=\int_{\mathcal{R}}\left(\varphi^{2}(1-\varphi)^{2}+\frac{\sigma}{2}|\operatorname{grad} \varphi|^{2}\right) \mathrm{d} v
$$

for the dimensionless Cahn-Hilliard eq. (59), which occurs as the volume occupied by the transition layers decreases. A plot of $\Psi_{\mathrm{CH}}$ versus the dimensionless time $t$ is provided in Fig. 1, from which it is apparent that a pronounced drop occurs at $t=t^{*}$.

A consequence of the natural boundary conditions (65) and the initial condition (68) is that any solution $\varphi$ of the dimensionless Cahn-Hilliard equation (59) must satisfy the condition

$$
\Delta \Phi_{\mathrm{CH}}=\frac{\int_{\mathcal{R}}\left(\varphi-c_{0}\right) \mathrm{d} v}{\int_{\mathcal{R}} c_{0} \mathrm{~d} v}=0,
$$

meaning, in view of the constraint (8), that the total constituent concentration must be conserved. To determine the extent to which our scheme satisfies the foregoing requirement, we use our numerically generated solution $\varphi$ to the initial-value problem for (70) and the initial condition (68) to compute $\Delta \Phi_{\mathrm{CH}}$ at each dimensionless time step. The resulting plot, provided in Fig. 2, shows that $\Delta \Phi_{\mathrm{CH}}$ is conserved to within a tolerance of $1.6 \times 10^{-7}$.

In Fig. 3, we plot $\varphi$ at dimensionless time $t=1.192$ on the line segment $\left\{\boldsymbol{x}:\left|x_{1}\right|<1, x_{2}=0, x_{3}=0\right\}$ along with the corresponding data from the benchmark simulations of Jeong et al. [24, Table 3]. Although the benchmark data is for the spherically symmetric version of the problem, we see reasonable agreement. Notice also that $\varphi$ takes values outside the interval $[0,1]$. As noted in the second paragraph at the beginning of Sect. 6 , this is a manifestation of certain inherently nonphysical features of the classical formulation of the Cahn-Hilliard equation. We next compare the Allen-Cahn type diffusion theory to our results for the Cahn-Hilliard theory.

\subsubsection{Allen-Cahn type diffusion theory}

In Figs. 4 and 5, we compare the profiles of $\varphi$ and $c$, respectively, on the line segment $\{\boldsymbol{x}$ : $\left.-1<x_{1}<1, x_{2}=0, x_{3}=0\right\}$ at dimensionless times $t=0.5, t=1.0, t=1.286$, and $t=1.5$, as determined

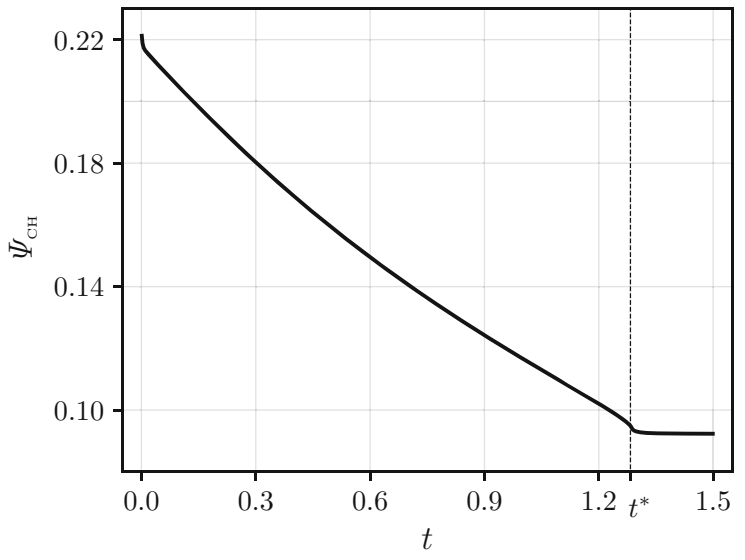

Fig. 1 The dimensionless total free-energy $\Psi_{\mathrm{CH}}$ defined in (75) versus the dimensionless time $t$. The dotted line indicates the critical time $t^{*}$ when the inner wall of the shell within which $\varphi=1$ collapses

by solving (71) subject to the initial condition determined by (69) and (73) for various values of $l$ to the analogous profiles obtained from solving (70) subject to the initial condition determined by (68) and (73). From these profiles, we see that, for the AllenCahn type diffusion theory, the transition layers of both $\varphi$ and $c$ move inward more rapidly than the corresponding layers for the Cahn-Hilliard theory but that this discrepancy diminishes noticeably as $l$ decreases. At the dimensionless times $t=0.5$ and $t=1.0$, Fig. 4 contains profiles of $\varphi$ with negative values in the collapsing region. This is a consequence of the particular choice of double-well potential (56), which, as in the Cahn-Hilliard case, does not penalize values of $\varphi$ outside of $[0,1]$. For this potential, the concentration $c$ is also not constrained to the interval $[0,1]$, as Fig. 5 shows. We also observe from Fig. 4 that, at $t=0.5$ and around $x_{1}=0, \varphi$ is negative, independent of $l$, and approximately constant. This suggests that $\triangle \varphi$ can be neglected around $x_{1}=0$ and hence, by $(62)_{2}$, that $v$ can be considered as an $l$ independent constant. Since $\varphi$ is negative and constant around $x_{1}=0$ and the same is true of $v$, we conclude from (63) that there, as indicated in Fig. 5, the greater the value of $l$ the more negative the corresponding value of $c$ must be. The same thing happens at $t=1.0$. With similar reasoning, it can thus be shown that the opposite occurs around $x_{1}=0$ at $t=1.5$ : the greater the value of $l$ the more positive the corresponding value $c$ must be. 


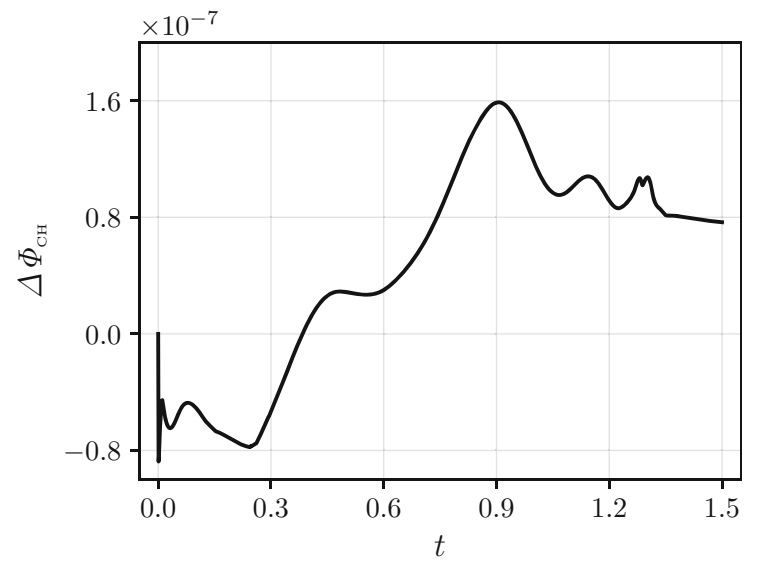

Fig. 2 The dimensionless measure $\Delta \Phi_{\mathrm{CH}}$ of constituent conservation defined in (76) versus the dimensionless time $t$, from which it follows that $\left|\Delta \Phi_{\mathrm{CH}}\right| \leq 1.6 \times 10^{-7}$

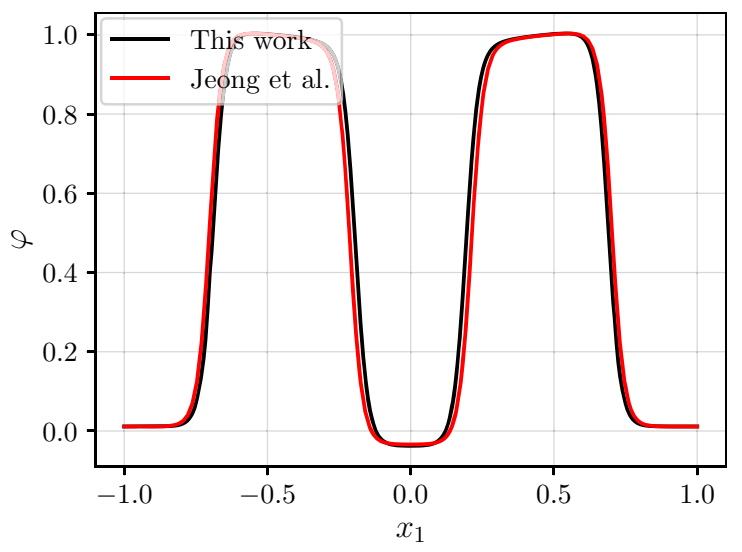

Fig. 3 Profiles on the line segment $\left\{\boldsymbol{x}:\left|x_{1}\right|<1, x_{2}=0\right.$, $\left.x_{3}=0\right\}$, of the phase field $\varphi$ at dimensionless time $t=1.192$ determined by solving the initial-boundary value problem for the Cahn-Hilliard theory (black) and the corresponding data (red) from the benchmark simulations of Jeong et al. [24, Table 3]

In Fig. 6, we plot the averages distances $r_{o}$ and $r_{i}$ to the intersections between the outer and inner portions of the level surface $\{\boldsymbol{x}: \varphi(\boldsymbol{x}, 0) \approx 1 / 2\}$ and the line segment $\left\{\boldsymbol{x}:\left|x_{1}\right|<1, x_{2}=0, x_{3}=0\right\}$. In that figure, dotted and solid are used to distinguish $r_{o}$ and $r_{i}$, respectively. Consistent with the results obtained in our solution of the initial-value problem for (70) and, thus, with the benchmark results, the inner transition layer accelerates inward as $t$ increases and the roughly spherical inclusion where $\varphi \approx 0$ eventually collapses and the inner transition layer disappears. The velocity of outer transition layer decreases shortly prior to the disappearance of the inclusion and vanishes subsequently as the system approaches equilibrium. In Table 1, we present the critical time $t_{l}^{*}$ and $r_{o}$ at the final time $t=1.5$ for different values of the coupling coefficient $l$ and for the Cahn-Hilliard theory (70). In Fig. 7, we plot $t_{l}^{*}$ and $r_{o}$ at $t=1.5$ along with the corresponding quantities from the Cahn-Hilliard theory, which are indicated by dotted horizontal lines. The figures show again that the approximation to the results for the Cahn-Hilliard theory afforded by the Allen-Cahn type diffusion theory improves as the dimensionless coupling energy coefficient $l$ defined in $(57)_{2}$ decreases.

We hereafter use $\varphi_{l}$ and $v_{l}$ to denote our numerical solution to the initial-boundary value problem for the Allen-Cahn type diffusion theory and thereby distinguish these quantities from the phase field $\varphi$ and the chemical potential $v$ obtained from the corresponding problem for the Cahn-Hilliard theory. In Fig. 8, we make use of the alternative representation (64) of $\psi_{\mathrm{AC}}$ to calculate and plot the total dimensionless freeenergy

$$
\begin{aligned}
\Psi_{\mathrm{AC}}= & \int_{\mathcal{R}} \varphi_{l}^{2}\left(1-\varphi_{l}\right)^{2} \mathrm{~d} v \\
& +\int_{\mathcal{R}}\left(\frac{\sigma}{2}\left|\operatorname{grad} \varphi_{l}\right|^{2}+\frac{l}{2} v_{l}^{2}\right) \mathrm{d} v
\end{aligned}
$$

for the Allen-Cahn type diffusion system (62) versus time for various values of the coupling coefficient $l$. Consistent with thermodynamic requirements, our results show that $\Psi_{\mathrm{AC}}$ decays monotonically for all values of $l$ considered. Moreover, comparison to Fig. 1 demonstrates that the deviation between $\Psi_{\text {AC }}$ and $\Psi_{\mathrm{CH}}$ decreases monotonically as $\imath$ decreases.

In Fig. 9, we plot the normalized difference

$$
\Delta \Phi_{\mathrm{AC}}=\frac{\int_{\mathcal{R}}\left(\varphi-\varphi_{l}\right) \mathrm{d} v}{\int_{\mathcal{R}} \varphi \mathrm{d} v}
$$

between the total values of the phase field for the solutions of the initial-value problems for (70) and (71) versus the dimensionless time for various values of the dimensionless coupling coefficient. In contrast to $\Delta \Phi_{\mathrm{CH}}$ defined in (76), $\Delta \Phi_{\mathrm{AC}}$ increases with dimensionless time for each value of the dimensionless coupling energy coefficient $l$ considered. From this, 

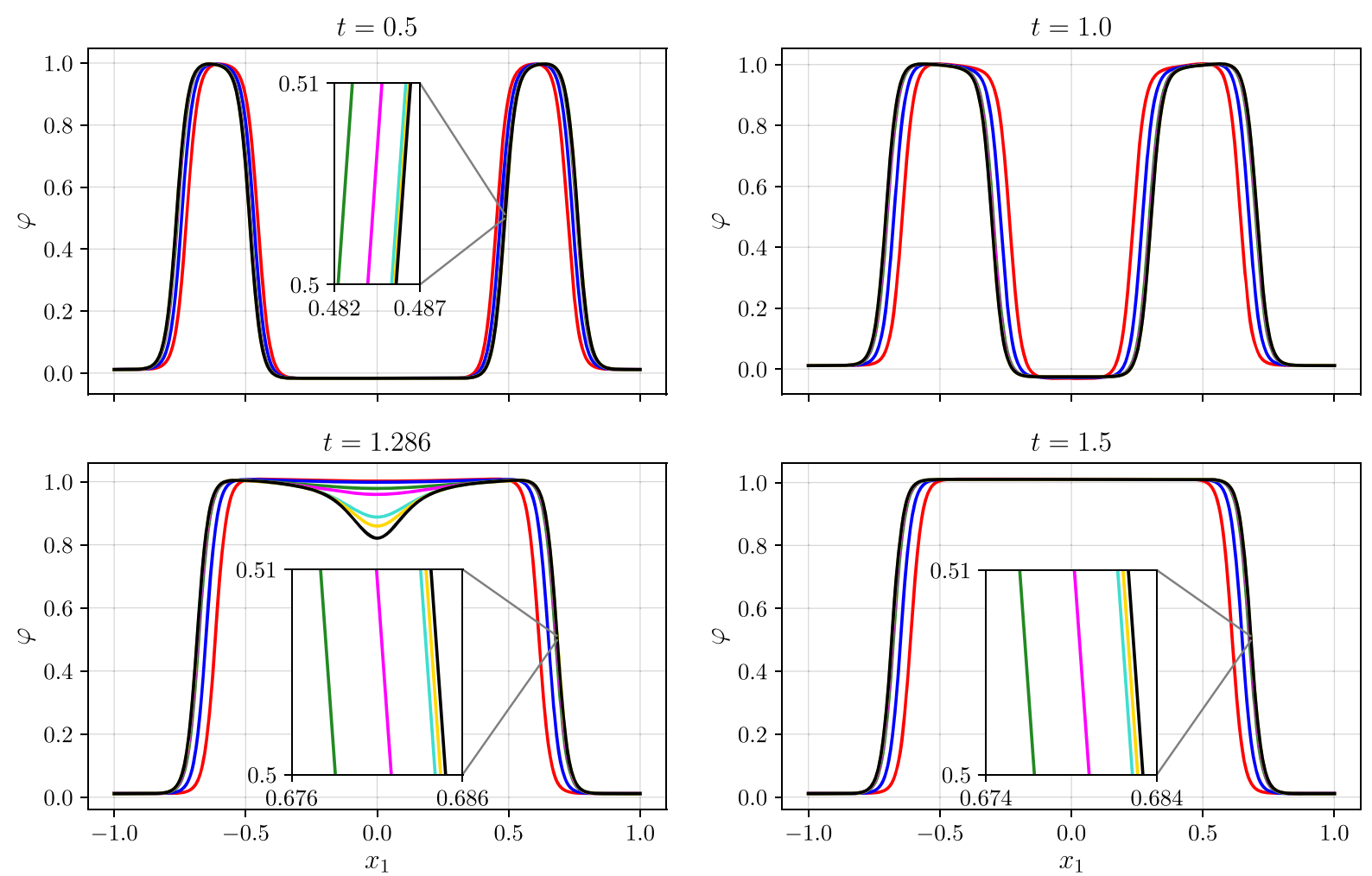

$$
\left[\begin{array}{l}
\iota=8.0 \times 10^{0} \\
\hdashline=4.0 \times 10^{0}
\end{array} \quad \begin{array}{l}
\iota=8.0 \times 10^{-1} \\
\iota=4.0 \times 10^{-1}
\end{array} \quad \begin{array}{l}
\iota=8.0 \times 10^{-2} \\
\iota=4.0 \times 10^{-2}
\end{array} \quad-\mathrm{CH}\right.
$$

Fig. 4 Profiles, at dimensionless times $t=0.5, t=1.0$, $t=1.286$, and $t=1.5$, of the phase field $\varphi$ on the line segment $\left\{\boldsymbol{x}:-1<x_{1}<1, x_{2}=0, x_{3}=0\right\}$ for various values of $l$ and the analogous profiles obtained from solving (70) subject to the initial condition determined by (68) and (73). These profiles

we see that the volume of the shell in the Allen-Cahn type diffusion system (62) decreases as $l$ increases. However, the growth of $\Delta \Phi_{\mathrm{AC}}$ with $t$ decreases monotonically as $\imath$ decreases.

To study the convergence of the results for the Allen-Cahn type diffusion theory towards those of the Cahn-Hilliard theory, we compute the time-averaged $L^{2}$ error

$e\left(u, u_{l}\right)=\sqrt{\frac{1}{t_{f}-t_{i}} \int_{t_{i}}^{t_{f}} \int_{\mathcal{R}}\left|u-u_{l}\right|^{2} \mathrm{~d} v \mathrm{~d} t}$,

for $\quad\left(u, u_{l}\right)=\left(\varphi, \varphi_{l}\right), \quad\left(u, u_{l}\right)=\left(\varphi, \varphi_{l}+i v_{l}\right)$, $\left(u, u_{l}\right)=\left(\psi_{\mathrm{AC}}, \psi_{\mathrm{CH}}\right)$, and $\left(u, u_{l}\right)=\left(v, v_{l}\right)$, bearing in mind the internal constraint $c=\varphi$ that applies in the Cahn-Hilliard theory. From plots of these quantities provided in Fig. 10, we see that $\varphi_{l}, \varphi_{l}+i v_{l}$, and $\psi_{\mathrm{AC}}$ show that the approximation to the results for the Cahn-Hilliard theory afforded by the Allen-Cahn type diffusion theory improves as the dimensionless coupling energy coefficient $l$ defined in $(57)_{2}$ decreases

converge linearly with $\imath$ to $\varphi, \varphi$, and $\psi_{\mathrm{CH}}$. In Fig. 11, we see that the chemical potential $v_{l}$ converges to $v$ as $l \rightarrow 0$, albeit at a rate considerably slower than linear. We speculate that the key to developing improved numerical strategies for solving the Allen-Cahn type diffusion theory might hinge on finding ways to reduce the error incurred in approximating the chemical potential of the Cahn-Hilliard theory. This difficulty likely stems from the nonlocal nature of that quantity.

\section{Discussion and conclusions}

In this paper, we formulated two continuum theories, one constrained and the other unconstrained, for constituent migration in bodies with microstructure described by a scalar phase field. The theories are built 

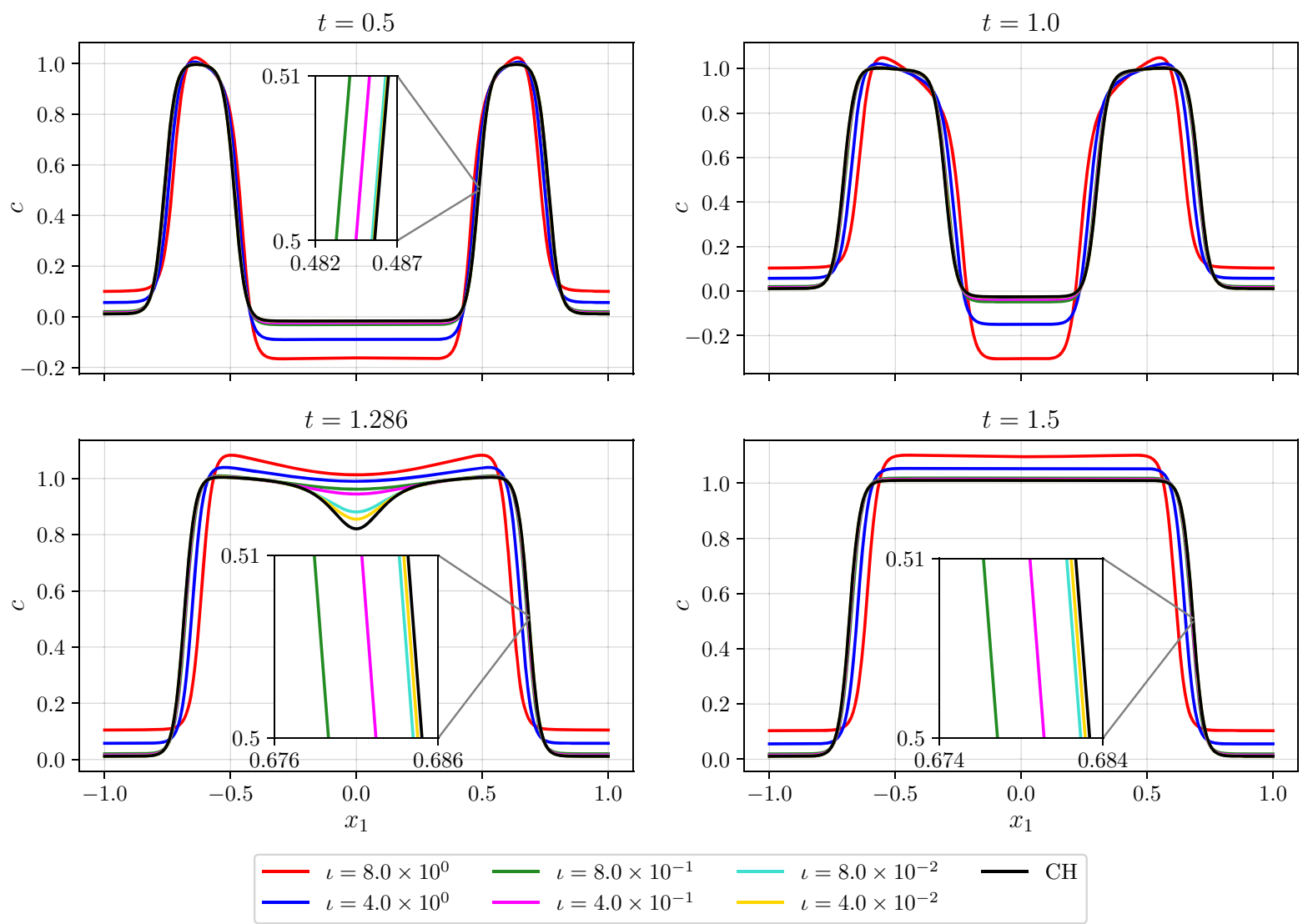

Fig. 5 Profiles, at dimensionless times $t=0.5, t=1.0$, $t=1.286$, and $t=1.5$, of the constituent concentration $c=$ $\varphi+i v$ on the line segment $\left\{\boldsymbol{x}:-1<x_{1}<1, x_{2}=0, x_{3}=0\right\}$ for various values of $l$ determined from (63) by postpocessing after obtaining $\varphi$ and $v$ by solving (71) subject to the initial condition

on the same basic principles-namely the constituent content balance, the microforce balance, and the freeenergy imbalance-but rely on different constitutive assumptions. In the constrained theory, the concentration and phase field are constrained to coincide, whereas in the unconstrained theory they are considered independent. From these alternative approaches, we provided a new derivation of the Cahn-Hilliard equation. On the basis of that derivation, we found that the Cahn-Hilliard equation can be interpreted as the limiting variant of a system of Allen-Cahn type diffusion equations that arises from the unconstrained theory. We then used numerical simulations of a benchmark problem proposed recently by Jeong et al. [24] to support this new interpretation. In particular, we found through an error comparison based on varying the dimensionless coupling energy coefficient determined by (68) and (73). As with the profiles of the phase field $\varphi$ presented in Fig. 4, these profiles show an approximation to the results for the Cahn-Hilliard theory that improves as the dimensionless coupling energy coefficient $l$ defined in $(57)_{2}$ decreases

that the numerical results of the Allen-Cahn type diffusion system converge linearly to those of the Cahn-Hilliard equation as the coupling coefficient tends to zero.

In the constrained theory developed here, the chemical potential and the internal microforce density are decomposed into sums of reactive and active components. As a consequence of requirement that the reactions be powerless, it follows that they must be equal and, moreover, that only the difference between the actions can be assigned constitutively. It is consequently permissible to prescribe the form the active component of the chemical potential. If, in particular, we choose that quantity to vanish identically, then the chemical potential is necessarily a pure reaction which, consistent with intuition, serves to 


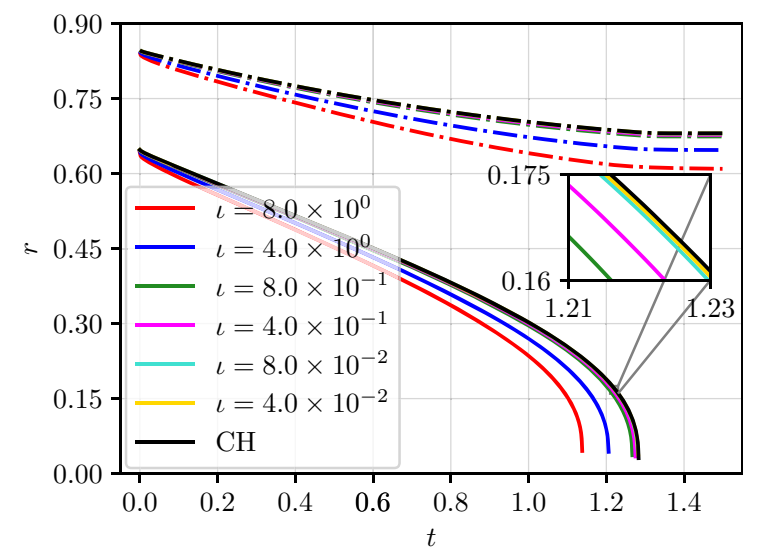

Fig. 6 Average of the distances $r_{o}$ and $r_{i}$ at which the outer (dotted lines) and inner (solid lines) transition layers intersect the line segment $\left\{\boldsymbol{x}:\left|x_{1}\right|<1, x_{2}=0, x_{3}=0\right\}$ versus the dimensionless time $t$. The inner transition layer accelerates inward until the collapse of the roughly spherical inclusion. The outer transition layer moves inward at a decreasing velocity and appears to come to rest after the inner layer disappears

Table 1 Critical time $t_{l}^{*}$ and the average distance $r_{o}>0$ of the intersections between the outer portion of the level surface $\{\boldsymbol{x}: \varphi(\boldsymbol{x}, 0) \approx 1 / 2\}$ and the line segment $\left\{\boldsymbol{x}:\left|x_{1}\right|<1, x_{2}=\right.$ $\left.0, x_{3}=0\right\}$ at the final time $t=1.5$ for the Allen-Cahn type diffusion system (71) with various values of the coupling coefficient $l$ and for the Cahn-Hilliard theory (70) denoted by $\mathrm{CH}$.

\begin{tabular}{lll}
\hline$l$ & $t_{i}^{*}$ & $r_{o}$ at $t=1.5$ \\
\hline $8.0 \times 10^{0}$ & 1.139 & 0.609475586517 \\
$4.0 \times 10^{0}$ & 1.207 & 0.647080424181 \\
$8.0 \times 10^{-1}$ & 1.267 & 0.674244203927 \\
$4.0 \times 10^{-1}$ & 1.275 & 0.677421146421 \\
$8.0 \times 10^{-2}$ & 1.282 & 0.679920378295 \\
$4.0 \times 10^{-2}$ & 1.282 & 0.680229937614 \\
$\mathrm{CH}$ & 1.283 & 0.680565172583 \\
\hline
\end{tabular}

For each choice of $l$, the critical time $t_{l}^{*}$ underestimates the value 1.283 obtained for the Cahn-Hilliard theory but the discrepancy decreases as $\imath$ decreases

ensure that, granted no-flux boundary conditions, the total concentration is preserved.

To conclude, we remark on the commonalities and differences between our derivation of the CahnHilliard equation and the derivation originated by Gurtin [3]. These derivations share the same basic principles and are predicated on the assumption that the phase field coincides with the constituent

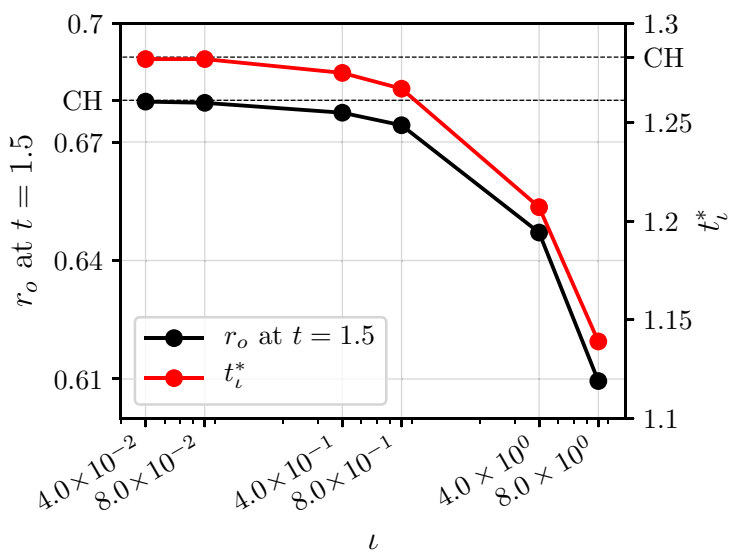

Fig. 7 Plot of the critical time $t_{t}^{*}$ and the average distance $r_{o}>0$ versus the dimensionless coupling energy coefficient $l$. The critical time $t_{t}^{*}$ is defined as the time at which the inner transition layer of the roughly spherical shell in which $\varphi=1$ collapses. The value $r_{o}>0$ is the average distance to the intersections between the outer portion of the level surface $\{\boldsymbol{x}$ : $\varphi(\boldsymbol{x}, 0) \approx 1 / 2\}$ and the line segment $\left\{\boldsymbol{x}:\left|x_{1}\right|<1, x_{2}=0, x_{3}=\right.$ $0\}$ at the final time $t=1.5$ of the simulation

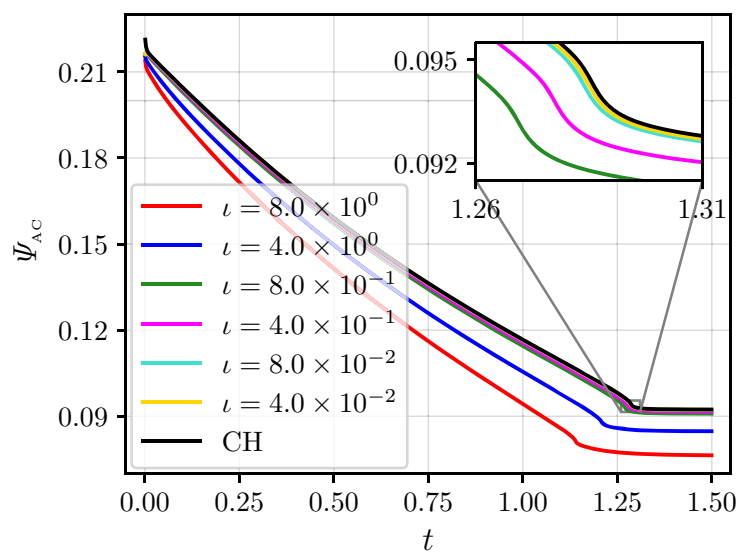

Fig. 8 The dimensionless total free-energy $\Psi_{\mathrm{AC}}$ defined in (77) versus the dimensionless time $t$ for various values of the dimensionless coupling energy coefficient $l$. This quantity decreases monotonically for all values of $l$ until the collapse of the inner transition layer, when it exhibits a sudden drop. The corresponding quantity, $\Psi_{\mathrm{AC}}$, as defined in (75), for the CahnHilliard theory is also shown

concentration. Their essential difference lies with the approach to treating the mentioned coincidence. Whereas Gurtin [3] identifies the concentration with the phase field from the outset, we treat the equality between concentration and phase field as an internal constraint that must be maintained by suitable reactions. Our motivation for doing that stems from the 


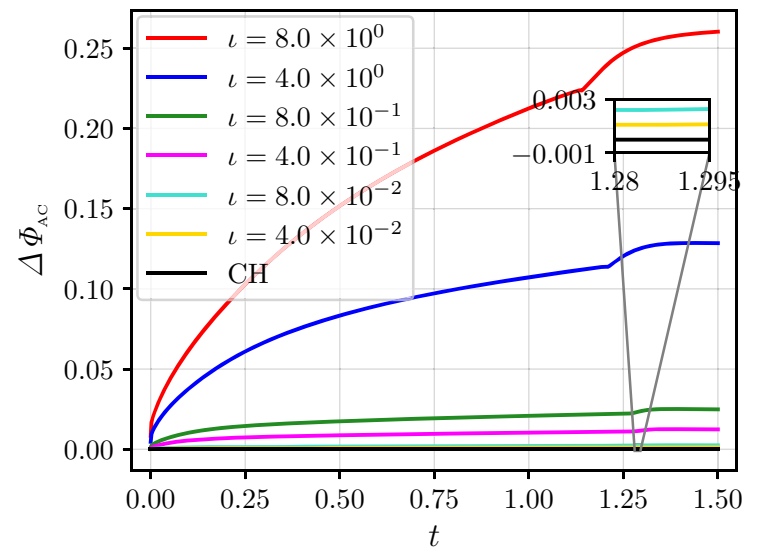

Fig. 9 Plot of the relative difference $\Delta \Phi_{\mathrm{AC}}$ of the total constituent versus the dimensionless time $t$ for various values of the dimensionless coupling energy coefficient $l$. Positive values of $\Delta \Phi_{\mathrm{AC}}$ indicate that the total concentration of the solution arising from the Cahn-Hilliard theory is greater than that arising from the Allen-Cahn type diffusion theory. The difference $\Delta \Phi_{\mathrm{AC}}$ reduces monotonically as $\imath$ decreases, indicating that the approximation provided by latter theory improves as $\iota \rightarrow 0$

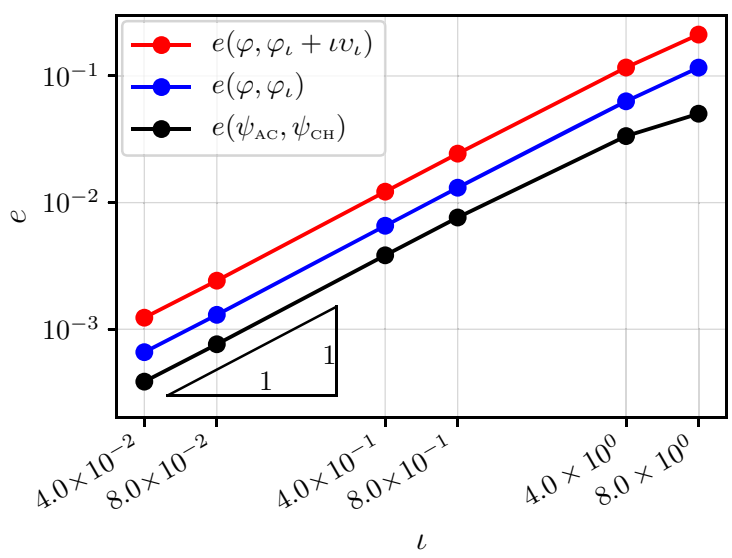

Fig. 10 Plot of the cumulative errors $e\left(\varphi, \varphi_{l}\right), e\left(\varphi, \varphi_{l}+w_{l}\right)$ and $e\left(\psi_{\mathrm{AC}}, \psi_{\mathrm{CH}}\right)$ of the phase field $\varphi$, concentration $c$, and freeenergy density $\psi$, as determined by (79), versus the dimensionless coupling energy coefficient $l$ defined in $(57)_{2}$. Each of these quantities decreases linearly with $l$ as $l \rightarrow 0$

prominent importance of internally constrained materials in continuum mechanics and the consequential belief that the explicit recognition of internal constraints that tacitly underpin certain phase-field models can provide important insights opening new possibilities that may deserve further investigation.

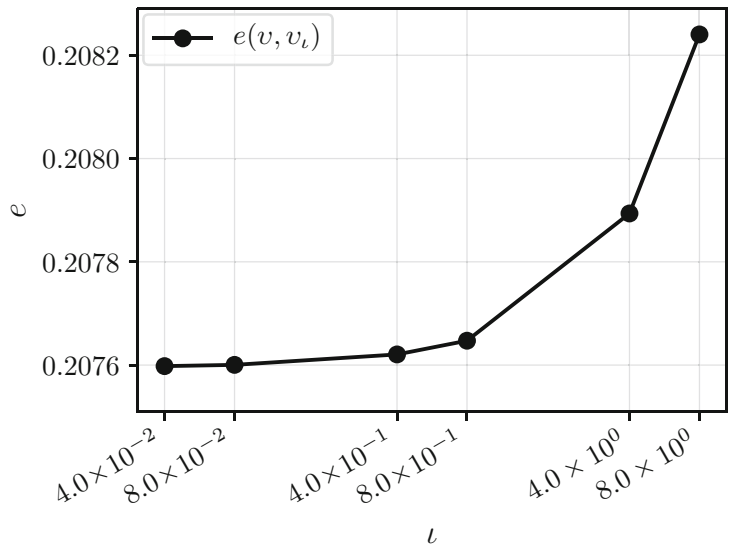

Fig. 11 Plot of the cumulative error $e\left(v, v_{l}\right)$ of the chemical potential $v$ determined by (79), versus the dimensionless coupling energy coefficient $l$ defined in $(57)_{2}$. The rate at which this error decreases as $l \rightarrow 0$ is much slower than the linear rate observed for the quantities considered in Fig. 10

Acknowledgements F.P. Duda gratefully acknowledges the support provided by the Brazilian agency $\mathrm{CNPq}$ and thanks the Okinawa Institute of Science and Technology for sponsoring a visit during which this work was conducted.

Funding This study was funded in part by $\mathrm{CNPq}$ through grant \#311587/2016-0 and by the Okinawa Institute of Science and Technology Graduate University with subsidy funding from the Cabinet Office, Government of Japan.

\section{Declarations}

Conflict of interest The authors declare that they have no conflict of interest.

Ethical standards The authors declare that they have complied with all ethical standards.

Open Access This article is licensed under a Creative Commons Attribution 4.0 International License, which permits use, sharing, adaptation, distribution and reproduction in any medium or format, as long as you give appropriate credit to the original author(s) and the source, provide a link to the Creative Commons licence, and indicate if changes were made. The images or other third party material in this article are included in the article's Creative Commons licence, unless indicated otherwise in a credit line to the material. If material is not included in the article's Creative Commons licence and your intended use is not permitted by statutory regulation or exceeds the permitted use, you will need to obtain permission directly from the copyright holder. To view a copy of this licence, visit http://creativecommons.org/licenses/by/4.0/. 


\section{References}

1. Cahn JW, Hilliard JE (1958) Free energy of a nonuniform system. I. Interfacial Free Energy. J Chem Phys 28(2):258-267. https://doi.org/10.1063/1.1744102

2. Allen SM, Cahn JW (1979) A microscopic theory for antiphase boundary motion and its application to antiphase domain coarsening. Acta Metall 27(6):1085-1095. https:// doi.org/10.1016/0001-6160(79)90196-2

3. Gurtin ME (1996) Generalized Ginzburg-Landau and Cahn-Hilliard equations based on a microforce balance. Phys D 92(3):178-192. https://doi.org/10.1016/01672789(95)00173-5

4. Miranville A (1999) A model of Cahn-Hilliard equation based on a microforce balance. C R Math Acad Sci Paris 328(12):1247-1252. https://doi.org/10.1016/S0764-4442(99) 80448-0

5. Fried E, Sellers S (2000b) Theory for atomic diffusion on fixed and deformable crystal lattices. J Elast 59(1):67-81. https://doi.org/10.1023/A:1011044929571

6. Podio-Guidugli P (2006) Models of phase segregation and diffusion of atomic constituent on a lattice. Ric Mat 55(1):105-118. https://doi.org/10.1007/s11587-006-0008-8

7. Morro A (2007) Phase-field models for fluid mixtures. Math Comput Modell 45(9):1042-1052. https://doi.org/10.1016/ j.mcm.2006.08.011

8. Heida M, Málek J, Rajagopal KR (2012) On the development and generalizations of Cahn-Hilliard equations within a thermodynamic framework. Z Angew Math Physik 63(1):145-169. https://doi.org/10.1007/s00033-011-0139-y

9. Duda FP, Ciarbonetti A, Sánchez PJ, Huespe AE (2015) A phase-field/gradient damage model for brittle fracture in elastic-plastic solids. Int J Plast 65:269-296. https://doi.org/ 10.1016/j.ijplas.2014.09.005

10. Duda FP, Ciarbonetti A, Toro S, Huespe A (2018) A phasefield model for solute-assisted brittle fracture in elasticplastic solids. Int J Plast 102:16-40. https://doi.org/10.1016/ j.ijplas.2017.11.004

11. da Silva MN, Duda FP, Fried E (2013) Sharp-crack limit of a phase-field model for brittle fracture. J Mech Phys Solids 61(11):2178-2195. https://doi.org/10.1016/j.jmps.2013.07. 001

12. Fried E, Gurtin ME (1993) Continuum theory of thermally induced phase transitions based on an order parameter. Phys D 68(3):326-343. https://doi.org/10.1016/0167-2789(93) 90128-N

13. Capriz G (1989) Continua with microstructure. Springer, Berlin. https://doi.org/10.1007/978-1-4612-3584-2

14. Fried E, Sellers S (2000a) Microforces and the theory of solute transport. Z Angew Math Physik (ZAMP) 51(5):732751. https://doi.org/10.1007/PL00001517

15. Fried E, Gurtin ME (2007) Thermomechanics of the interface between a body and its environment. Continuum Mech Thermodyn 19(5):253-271. https://doi.org/10.1007/s00161007-0053-x

16. Binder K, Frisch HL (1991) Dynamics of surface enrichment: a theory based on the Kawasaki spin-exchange model in the presence of a wall. Z für Phys B 84(3):403-418. https://doi.org/10.1007/BF01314015
17. Fischer HP, Maass P, Dieterich W (1997) Novel surface modes in spinodal decomposition. Phys Rev Lett 79:893-896. https://doi.org/10.1103/PhysRevLett.79.893

18. Kenzler R, Eurich F, Maass P, Rinn B, Schropp J, Bohl E, Dieterich W (2001) Phase separation in confined geometries: solving the Cahn-Hilliard equation with generic boundary conditions. Comput Phys Commun 133(2):139-157. https://doi.org/10.1016/S0010-4655(00)0 0159-4

19. Goldstein GR, Miranville A, Schimperna G (2011) A CahnHilliard model in a domain with non-permeable walls. Phys D 240(8):754-766. https://doi.org/10.1016/j.physd.2010. 12.007

20. Heida M (2013) On the derivation of thermodynamically consistent boundary conditions for the Cahn-HilliardNavier-Stokes system. Int J Eng Sci 62:126-156. https:// doi.org/10.1016/j.ijengsci.2012.09.005

21. Liu C, Wu H (2019) An energetic variational approach for the Cahn-Hilliard equation with dynamic boundary condition: Model derivation and mathematical analysis. Arch Ration Mech Anal 233(1):167-247. https://doi.org/10.1007/ s00205-019-01356-x

22. Fukao T (2016) Convergence of Cahn-Hilliard systems to the Stefan problem with dynamic boundary conditions. Asymptotic Anal 99(1-2):1-21. https://doi.org/10.3233/ ASY-161373

23. Colli P, Fukao T (2015) The Allen-Cahn equation with dynamic boundary conditions and mass constraints. Math Methods Appl Sci 38:3950-3967. https://doi.org/10.1002/ mma.3329

24. Jeong D, Choi Y, Kim J (2018) A benchmark problem for the two- and three-dimensional Cahn-Hilliard equations. Commun Nonlinear Sci Numer Simul 61:149-159. https:// doi.org/10.1016/j.cnsns.2018.02.006

25. Elliott CM, Garcke H (1996) On the Cahn-Hilliard equation with degenerate mobility. SIAM J Math Anal 27(2):404-423. https://doi.org/10.1137/S003614109426 7662

26. Novick-Cohen A (2008) The Cahn-Hilliard equation. In: Dafermos C, Pokorny M (eds) Handbook of differential equations: evolutionary equations, vol IV. North-Holland, Amsterdam, pp 201-228. https://doi.org/10.1016/S18745717(08)00004-2

27. Hughes T, Cottrell J, Bazilevs Y (2005) Isogeometric analysis: CAD, finite elements, NURBS, exact geometry and mesh refinement. Comput Methods Appl Mech Eng 194(39):4135-4195. https://doi.org/10.1016/j.cma.2004. 10.008

28. Chung J, Hulbert G (1993) A time integration algorithm for structural dynamics with improved numerical dissipation: the generalized- $\alpha$ method. J Appl Mech 60:371-375. https:// doi.org/10.1115/1.2900803

29. Sarmiento A, Espath L, Vignal P, Dalcin L, Parsani M, Calo VM (2018) An energy-stable generalized- $\alpha$ method for the Swift-Hohenberg equation. J Comput Appl Math 344:836-851. https://doi.org/10.1016/j.cam.2017.11.004

30. Gómez H, Calo VM, Bazilevs Y, Hughes TJ (2008) Isogeometric analysis of the Cahn-Hilliard phase-field model. Comput Methods Appl Mech Eng 197(49):4333-4352. https://doi.org/10.1016/j.cma.2008.05.003 
31. Vignal P, Collier N, Dalcin L, Brown D, Calo VM (2017) An energy-stable time-integrator for phase-field models. Comput Methods Appl Mech Eng 316:1179-1214. https:// doi.org/10.1016/j.cma.2016.12.017
Publisher's Note Springer Nature remains neutral with regard to jurisdictional claims in published maps and institutional affiliations. 